\title{
Spectroscopic and Structural Characterization of Water-Shared Ion Pairs in Aqueous Sodium and Lithium Hydroxide
}

\author{
Denilson Mendes de Oliveira,${ }^{\dagger}$ Aria J. Bredt, ${ }^{\dagger}$ Tierney C. Miller, ${ }^{\dagger}$ \\ Steven A. Corcelli, ${ }^{\ddagger}$ and Dor Ben-Amotz ${ }^{* \dagger}$ \\ ${ }^{\dagger}$ Department of Chemistry, Purdue University, West Lafayette, Indiana 47907, United States \\ $₫$ Department of Chemistry and Biochemistry, University of Notre Dame, Notre Dame, Indiana 46556, United States \\ *E-mail: bendor@purdue.edu \\ Supporting Information
}

\section{Table of Contents}

1. Thermodynamic analysis methods and results

2. Additional experimental Raman-MCR spectra

3. Additional AIMD simulation predictions 


\section{Temperature dependent thermodynamic analysis}

The value of $K_{0}$ and $b$ were obtained from the experimentally measured intensities of the normalized SC spectra at vibrational frequencies specified below. The spectra are those shown in Fig. 2 (A) and (C) of the parent manuscript and Fig. S2 of the next subsection. The intensities were measured in the vicinity of the local minima in the high temperature spectra shown in Fig. 2 (B) and (D) of the parent manuscript, at five separate frequency windows of $\sim 5.3 \mathrm{~cm}^{-1}$ width centered at $3397,3402,3407,3413$, and $3418 \mathrm{~cm}^{-1}$ for the $\mathrm{NaOH} \mathrm{SC}$ spectra, and $3460,3465,3471,3496$, and $3481 \mathrm{~cm}^{-1}$ for the LiOH SC spectra. The intensities $I$ (counts) at each of the above frequency windows were fit to a quadratic function of the total salt concentration, $I=I_{0}+a_{1} c_{T}+$ $a_{2} c_{T}^{2}$, from which the average values and standard deviations of $K_{0}$ and $b$ were determined using $K_{0}=-a_{1} / I_{0}$ and $b=a_{2} / a_{1}-2 a_{1} / I_{0}$. Note that the latter expressions were derived from a Taylor expansion of Eq. 2 in the parent manuscript, performed by first Taylor expanding the numerator and then factoring out $K_{0} c_{T}$ before re-expanding the entire remaining expression to third order in $c_{T}$.
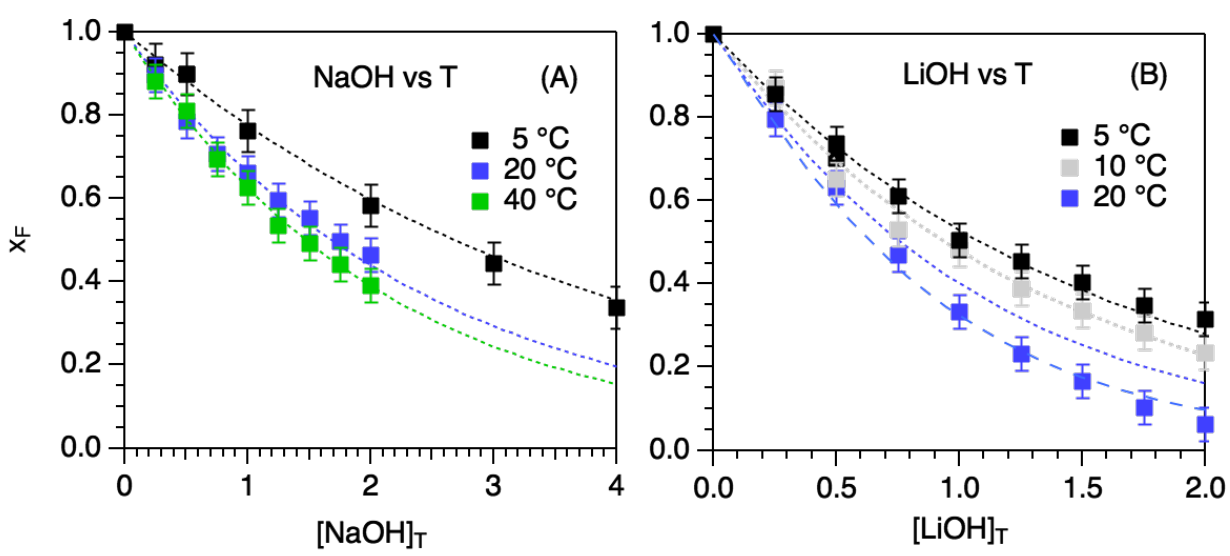

Figure S1. Dissociated ion pair fractions, $x_{F}=I / I_{0}$, were fit to obtain $K_{0}$ and $b$, as described above. The $5{ }^{\circ} \mathrm{C}$ points are the same as those in Fig. 3 (A) and (B) of the parent manuscript, and the points at other temperatures were obtained from the SC spectra shown in Fig. S2. The dotted curves are obtained using Eq. 2 with the $K_{0}$ and $b$ values in Table S1.

The dotted curves in Fig. S1 and Fig. 3 (A) and (B) of the parent manuscript, were obtained using Eq. 2 with the best fit $K_{0}$ and $b$ values given in Table S1. Note that all these predictions agree with the data points quite well, except for $\mathrm{LiOH}$ at $20^{\circ} \mathrm{C}$. This discrepancy may be linked to the fact that the dissociated ion pair fractions of $\mathrm{LiOH}$ at $20^{\circ} \mathrm{C}$ extended down to $x_{F} \sim 0.06$, 
while all the other results in Fig. S1 pertain to dissociated fractions $x_{F}>0.2$. The dashed blue curve in Fig. S1 (B) shows how the agreement with the predictions of Eq. 2 is improved if $K_{0} \sim 0.85$ is held fixed and $b \sim 2$ is obtained from a best fit to the blue $x_{F}$ points.

Table S1. $K_{0}$ and $b$ value obtained from the $x_{F}$ points shown in Fig. S1.

\begin{tabular}{|c|c|c|c|c|c|c|}
\hline & \multicolumn{3}{|c|}{$\mathrm{NaOH}$} & \multicolumn{3}{c|}{$\mathrm{LiOH}$} \\
\hline$T\left({ }^{\circ} \mathrm{C}\right)$ & $5{ }^{\circ} \mathrm{C}$ & $20{ }^{\circ} \mathrm{C}$ & $40{ }^{\circ} \mathrm{C}$ & $5{ }^{\circ} \mathrm{C}$ & $10{ }^{\circ} \mathrm{C}$ & $20{ }^{\circ} \mathrm{C}$ \\
\hline$K_{0}$ & $0.25 \pm 0.01$ & $0.41 \pm 0.03$ & $0.45 \pm 0.02$ & $0.61 \pm 0.02$ & $0.70 \pm 0.04$ & $0.85 \pm 0.03$ \\
\hline$b$ & $0.41 \pm 0.01$ & $0.64 \pm 0.04$ & $0.74 \pm 0.03$ & $1.00 \pm 0.04$ & $1.17 \pm 0.08$ & $1.47 \pm 0.06$ \\
\hline
\end{tabular}




\section{Additional Raman-MCR results}

Figure S2 shows concentration dependent Raman-MCR SC spectra of aqueous $\mathrm{NaOH}$ at $20{ }^{\circ} \mathrm{C}$ and $40{ }^{\circ} \mathrm{C}$ and aqueous $\mathrm{LiOH}$ at $10{ }^{\circ} \mathrm{C}$ and $20^{\circ} \mathrm{C}$. The corresponding results at $5{ }^{\circ} \mathrm{C}$ are shown in Fig. 2 (A) and (C) of the parent manuscript.
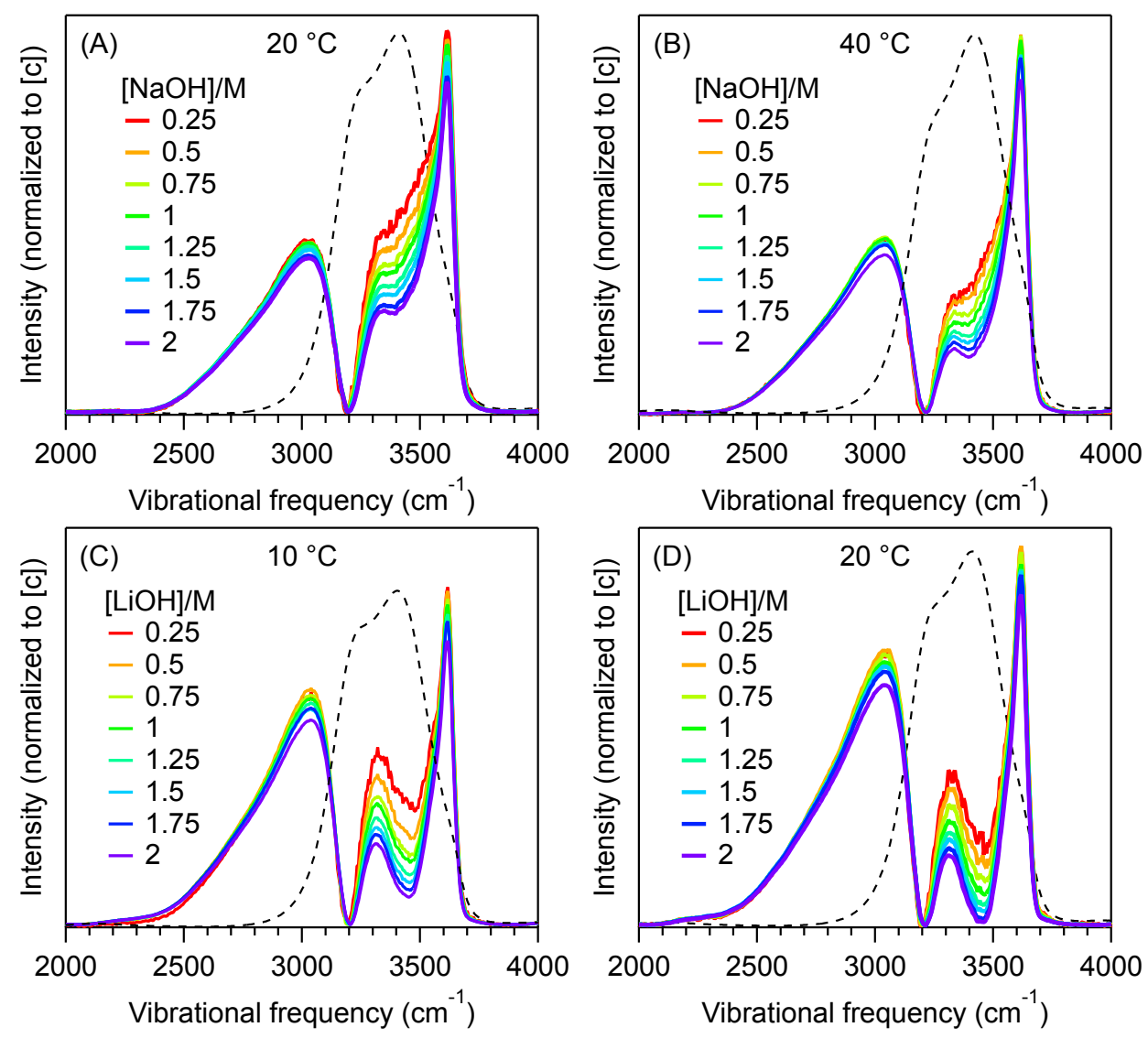

Figure S2. Concentration-dependent Raman-MCR hydration shell spectra of aqueous $\mathrm{NaOH}$ (A: and B: $40^{\circ} \mathrm{C}$ ) and $\mathrm{LiOH}\left(\mathrm{C}: 10^{\circ} \mathrm{C}\right.$ and $\mathrm{D}: 20^{\circ} \mathrm{C}$ ). The dashed curves are pure water spectra at the specified temperatures (scaled to approximately the same peak intensity).

All SC spectra in Figure S2 and Figure 2 of the parent manuscript were obtained after performing a background subtraction using a fourth-order polynomial fit to baseline points adjacent to the $\mathrm{OH}$ stretch band, as exemplified in Figure S3. This baseline fitting procedure facilitated the identification of the minimum area non-negative SC spectra as those SC spectra that 
have zero intensity (above background) at some point within the frequency range of the solvent (water) $\mathrm{OH}$ stretch band.
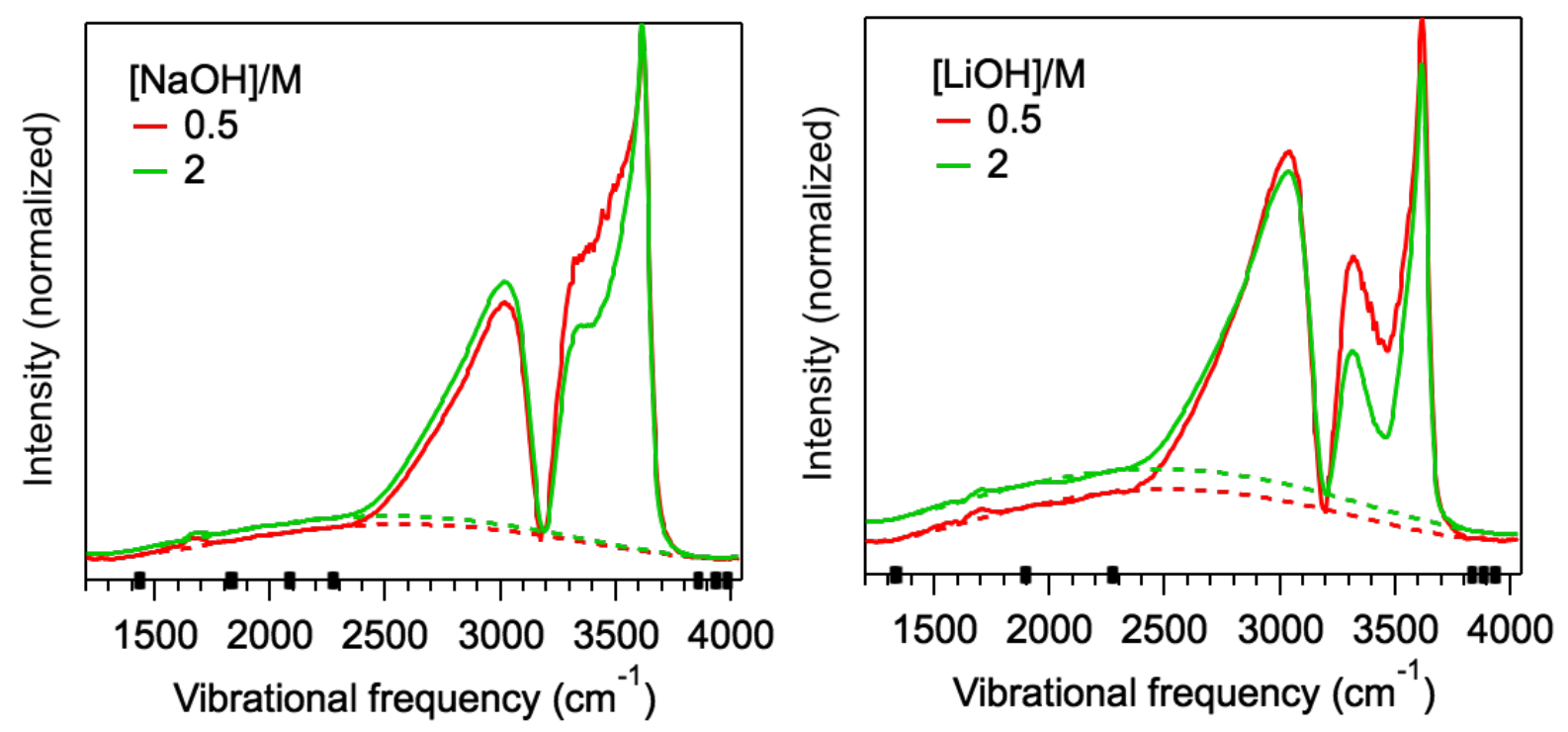

Figure S3. Raman-MCR SC spectra of aqueous $(0.5 \mathrm{M}$ and $2 \mathrm{M}) \mathrm{NaOH}$ and $\mathrm{LiOH}$ at $5{ }^{\circ} \mathrm{C}$ prior to background subtraction. The dashed curves represent the fourth-order polynomial function fit to the background points marked with black squares on the $x$-axis. The small peak near $1700 \mathrm{~cm}^{-1}$ arises from bend vibrations of water that are perturbed by $\mathrm{NaOH}$ or $\mathrm{LiOH}$. 


\section{Additional AIMD results}

Figure S4 shows AIMD results for the distribution of cations and water molecules with respect to the hydroxide ion. Specifically, the radial distribution functions, $g(r)$, and the respective numbers, $\mathrm{N}(\mathrm{r})$, of cations (upper two panels) and water oxygen atoms (lower two panels) are plotted with respect to their distance from the hydroxide oxygen atom.
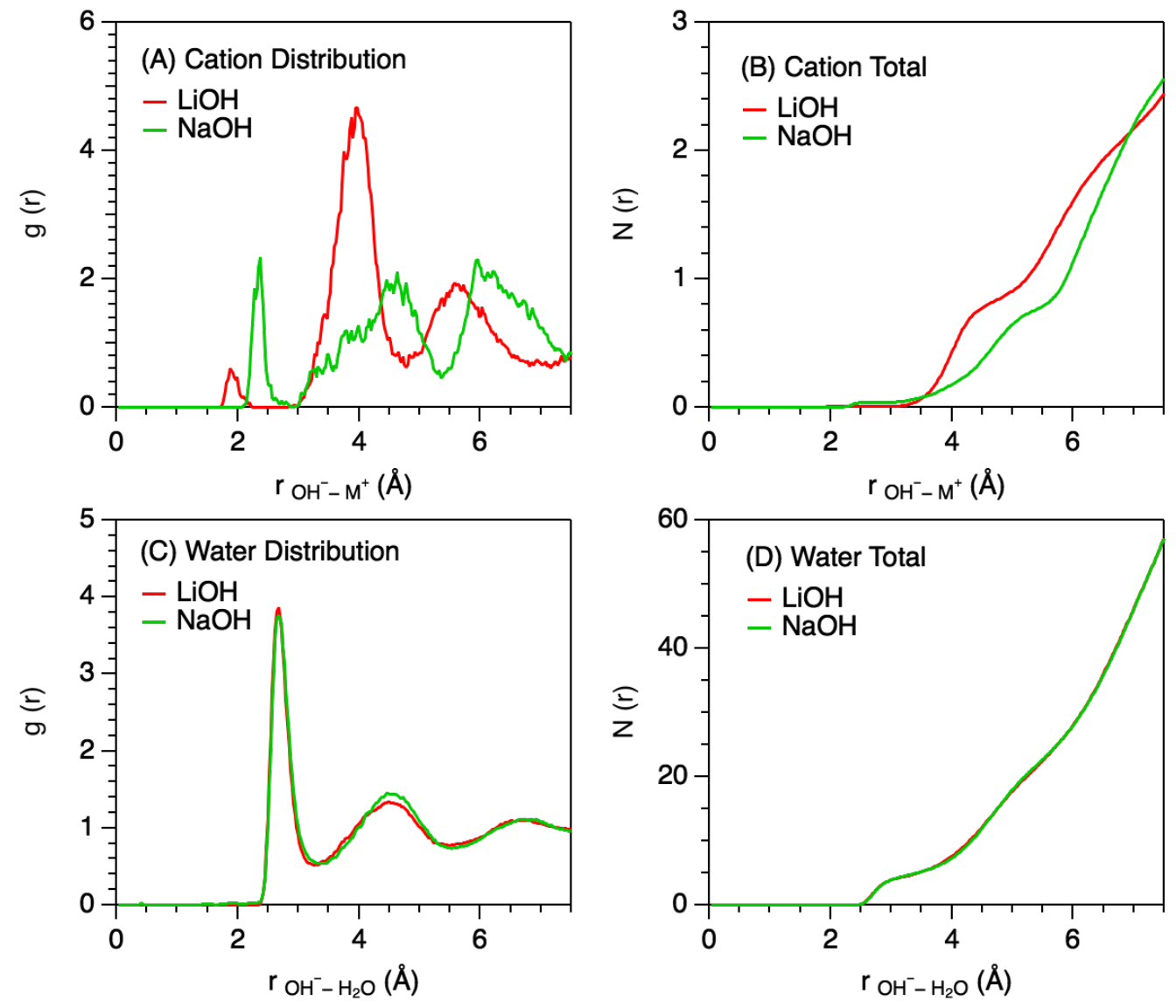

Figure S4. AIMD predictions of the populations of cations (A and B) or water molecules (C and D) surrounding the hydroxide ion in $1 \mathrm{M} \mathrm{LiOH}$ (red) and $1 \mathrm{M} \mathrm{NaOH}$ (green) at $25^{\circ} \mathrm{C}$. The radial distribution functions $\mathrm{g}(\mathrm{r})$ (A and $\mathrm{C}$ ) and integrated numbers of cations or water molecules N(r) ( $\mathrm{B}$ and $\mathrm{D}$ ) are plotted as a function of the distance between the $\mathrm{OH}^{-}$oxygen atom and either the cation, $\mathrm{M}^{+}$, or the oxygen atom of $\mathrm{H}_{2} \mathrm{O}$.

Figure S5 shows two dimensional plots of the distribution of water molecules around each hydroxide ion, plotted in the same way as the cation distribution functions shown in Fig. 1 (C) and 
(D) of the parent manuscript. The fact that these distributions are nearly identical confirms that the hydroxide hydration shell remains nearly fully intact even in the more significantly ion paired LiOH solution. Close inspection of the g(r) results in Fig. S4 (C) reveals that there are slightly fewer second hydration shell waters around $\mathrm{OH}^{-}$in the $\mathrm{LiOH}$ solution, as evidenced by the slightly smaller second $\mathrm{g}(\mathrm{r})$ peak in $\mathrm{LiOH}$ than $\mathrm{NaOH}$.
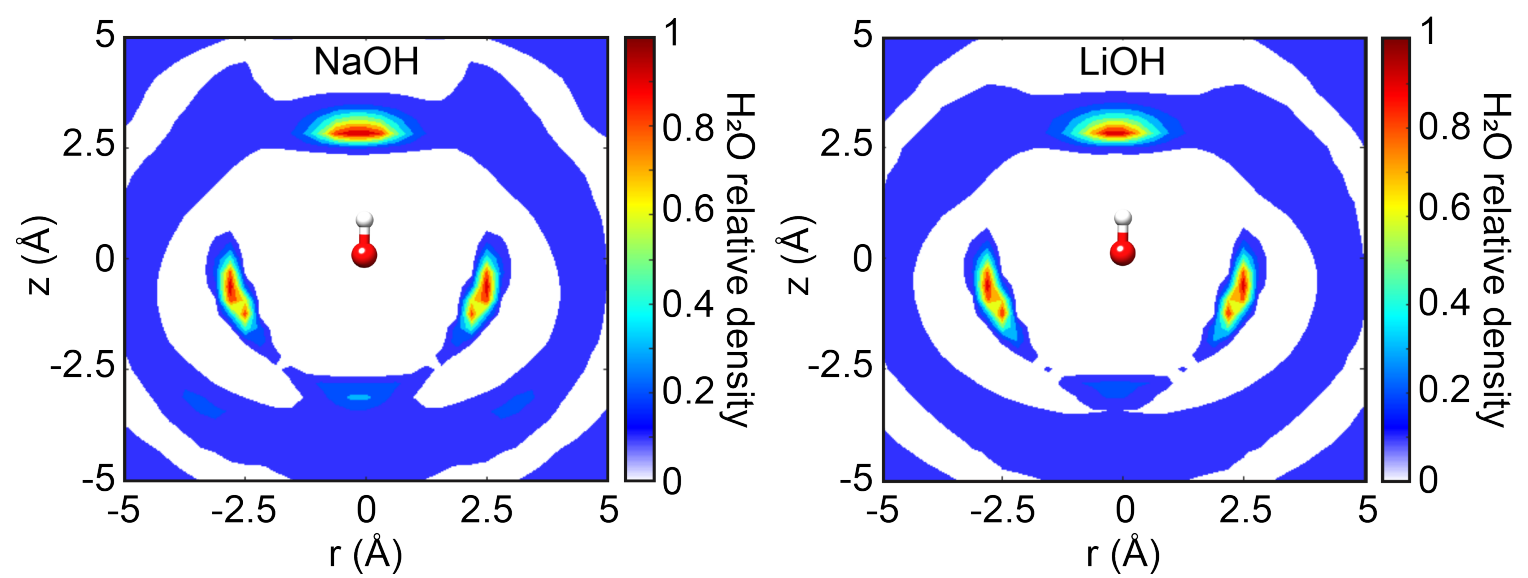

Figure S5. AIMD predictions of the distributions of $\mathrm{H}_{2} \mathrm{O}$ around $\mathrm{OH}^{-}$in $1 \mathrm{M}$ solutions of $\mathrm{NaOH}$ (right panel) and $\mathrm{LiOH}$ (left panel) at $25^{\circ} \mathrm{C}$. The $\mathrm{r}$ and $\mathrm{z}$ axes are distances relative to the hydroxide oxygen atom located at $(\mathrm{r}, \mathrm{z})=(0,0)$. 